J. Math. Kyoto Univ.

1-1 (1961) 87-99.

\title{
Complete reducibility of rational representations of a matric group
}

By

Masayoshi NaGATA

(Received July 6, 1961)

It is well known in the classical case that every rational representation of a semi-simple algebraic linear group is completely reducible. But the same argument becomes false in the case where the universal domain is of characteristic $p \neq 0$. For instance, when $K$ is a universal domain of characteristic 2, the simple group $S L(2, K)$ has the following rational representation $\rho$ which is not completely reducible :

$$
\rho\left(\begin{array}{ll}
a & b \\
c & d
\end{array}\right)=\left(\begin{array}{lll}
1 & a c & b d \\
0 & a^{2} & b^{2} \\
0 & c^{2} & d^{2}
\end{array}\right) .
$$

(This $\rho$ is not completely reducible because $a c$ and $b d$ are not linear polynomials in $a^{2}, b^{2}, c^{2}, d^{2}$.) Therefore it is an interesting question to ask conditions for an algebraic linear group $G$ so that every rational representation of $G$ is completely reducible.

The main purpose of the present paper is to answer the above question. We want to include algebraic groups in an algebraically closed field and also an arbitrary matric group over a field, and for that purpose, we use the following terminology.

A universal domain $K$ is defined to be just an algebraically closed field. An algebraic linear group with a universal domain $K$ is defined to be the set of $K$-rational points of an algebraic linear group in the usual sense, defined over a subfield of $K$ and with a universal domain (in the usual sense) which contains the field $K$. Let $K$ be a universal domain and let $G$ be a subgroup 
of $G L(n, K)$. The closure $G^{*}$ of $G$ in the Zariski topology on $G L(n, K)$ is again a group, hence is algebraic group. This $G^{*}$ is called the closure of $G$. A matric group $G$ is called connected if the closure $G^{*}$ of $G$ is connected. Let $G^{*}$ be the closure of a matric group $G$ and let $G_{0}^{*}$ be the connected component of the identity of $G^{*}$. Then $G_{0}^{*} \cap G$ is the largest connected subgroup of $G$, the closure of $G_{0}^{*} \cap G$ is $G_{0}^{*}$ and the indices $\left[G^{*}: G_{0}^{*}\right]$ and $\left[G:\left(G_{0}^{*} \cap G\right)\right]$ are equal to each other. This subgroup $G_{0}^{*} \cap G$ is called the connected component of the identity of $G$. A rational representation of a matric group $G$ is defined to be the restriction on $G$ of a rational representation of the closure of $G$.

In the following, let $K$ be a universal domain of characteristic $p$ which may or may not be zero, and let $G$ be a matric group contained in $G L(n, K)$.

Now, our main result, which is an answer of the above question, can be stated as follows:

(1) When $p \neq 0$ : Every rational representation of $G$ is completely reducible if and only if there is a normal subgroup $G_{0}$ of finite index such that (i) $G_{0}$ is a subgroup of a torus group (i.e., diagonalizable) and (ii) the index of $G_{0}$ in $G$ is prime to p. If $G$ is connected, then the above condition is equivalent to the condition that the representation of $G$ by homogeneous forms of degree $p$ is completely reducible. On the other hand, if $G$ is an algebraic group (which may not be connected), then the complete reducibility of all rational representations of $G$ is equivalent to the condition that every element of $G$ is semi-simple (i.e., diagonalizable).

(2) When $p=0$ : Each of the following two conditions is equivalent to the complete reducibility of all rational representations of $G$.

(I) The closure of $G$ has a faithful rational representation which is completely reducible.

(II) The radical of the closure of $G$ is a torus group.

We shall prove also the following interesting theorem concerning the complete reducibility of rational representations of a connected algebraic linear group :

If $G$ is a connected algebraic linear group, then every rational 
representation of $G$ is completely reducible if (and only if) the following is true:

If $\rho^{\prime}=\left(\begin{array}{ll}1 & \tau \\ 0 & \rho\end{array}\right)$ is a rational representation of $G$, then $\rho^{\prime}$ is equivalent to the representation $\left(\begin{array}{ll}1 & 0 \\ 0 & \rho\end{array}\right)$.

Some fundamental results on algebraic linear groups, which are contained in A. Borel's paper "Groupes linéaires algébriques", Ann. of Math. 64, No. 1 (1956), pp. 20-82, are used freely or with words "cf. Borel's paper" only.

\section{Preliminaries on connected algebraic linear groups.}

Let $K$ be a universal domain of an arbitrary characteristic and let $G$ be a connected algebraic linear group contained in $G L(n, K)$, throughout this section, except for in Lemma 4 .

A Borel subgroup $B$ of $G$ is defined to be a maximal connected solvable subgroug of $G$. Then as was proved by Borel, the following is true :

Lemma 1. The homogeneous variety $G / B$ is a projective variety. On the other hand, every element of $G$ is in some conjugate of $B$.

Now we have:

Lemma 2. If $u \in G$ is unipotent, then there is a closed connected unipotent subgroup of $G$ which contains $u$.

Proof. By the last half of Lemma 1, we see that $u$ is in a Borel subgroup $B$ of $G$. Since $B$ is solvable, the set $U$ of all unipotent elements of $B$ is a closed connected subgroup, which proves the assertion.

On the other hand, the following was proved by Borel:

Lemma 3. If $G$ consists merely of semi-simple elements, then $G$ is commutative, hence is a torus group.

Next we shall concern with an algebraic group which is not connected :

Lemma 4. Let $G$ be an algebraic linear group and let $G_{0}$ be 
the connected component of the identity of $G$. Then each coset $G_{0} g$ $(g \in G)$ contains an element of finite order.

Proof. Let $A$ be the smallest algebraic group containing $g$ and let $A_{0}$ be the connected component of the identity of $A$. Since $A$ is commutative and since $A_{0}$ is infinitely divisible (in the additive formulation), $A_{0} g$ contains an element of finite order ${ }^{1}$, which proves the assertion.

\section{Preliminaries on group representations.}

Let $G$ be an abstract group, let $G_{0}$ be a normal subgroup of $G$ and let $K$ be a field of characteristic $p$ which may be zero, throughout this section, except for in Lemmas 8 and 9.

The following lemma is well known : ${ }^{2}$

Lemma 5. If a finite $K$-module $M$ is a simple $K$-G-module, then $M$ is the direct sum of a finite number of $K$ - $G_{0}$-modules which are simple.

Corollary. If a representation $\rho$ of $G$ in $G L(n, K)$ is completely reducible, then the restriction of $\rho$ on $G_{0}$ is completely reducible.

The converse of the above corollary is not true in general if $p \neq 0$, but we have:

Lemma 6. ${ }^{33}$ Let $\rho$ be a representation of $G$ in $G L(n, K)$. If the restriction $\rho_{0}$ of $\rho$ on $G_{0}$ is completely reducible and if the index $t=\left[G: G_{0}\right]$ is finite and not divisible by $p$, then $\rho$ itself is completely reducible.

Proof. If $\rho$ is not completely reducible, then $\rho$ contains a representation of the form $\left(\begin{array}{ll}\rho_{1} & \tau \\ 0 & \rho_{2}\end{array}\right)$ which is not completely reducible and such that $\rho_{1}, \rho_{2}$ are irreducible. Hence we may assume

1) One can prove furthermore that $A=A_{0} \times F$ with a finite group $F$.

2) This is a special case of the following easy lemma in group theory: Let a group $\Gamma$ be an operator domain on a group $G$ and let $\Gamma^{\prime}$ be a normal subgroup of $\Gamma$. If $\Gamma^{\prime}$ contains all inner automorphisms of $G$, if $G$ is a simple $\Gamma$-group and if $G$ satisfies the maximum and minimum conditions for $\Gamma^{\prime}$-subgroups, then $G$ is the direct product of a finite number of $\Gamma^{\prime}$-simple subgroups.

3) The writer was informed by Dr. H. Matsumura that this lemma was given by C. Chevalley. 
that $\rho=\left(\begin{array}{cc}\rho_{1} & \tau \\ 0 & \rho_{2}\end{array}\right)$ and that $\rho_{1}, \rho_{2}$ are irreducible. Let the representation module of $\rho$ be $M^{*}$. $M^{*}$ contains the representation module $M$ of $\rho_{2}$, and $M^{*} / M$ is the representation module of $\rho_{1}$. Since $\rho_{0}$ is completely reducible, we see that $M$ is a direct summand of $M^{*}$ as a $G_{0}-K$-module. Hence $M^{*}=M \oplus N_{1} \oplus \cdots \oplus N_{r}$, where $N_{i}$ are simple $G_{0}-K$-modules. For each $N_{i}$, we fix a linearly independent basis $a_{i 1}, \cdots, a_{i s}$ over $K$; we note here that the number $s$ is independent of $i$ because $M^{*} / M$ is a simple $G-K$-module (remember the well known proof of Lemma 5). For each $(r, s)$. matrix $b=\left(b_{i j}\right)$ over the module $M$, we define $N(b)=\sum_{i j}\left(a_{i j}+b_{i j}\right) K$. Thus we have a one-one correspondence between all of $b$ and all of submodules $N$ such that $M^{*}=M \oplus N$ as an $K$-module ${ }^{4}$. We may assume, on the other hand, that $\rho_{1}$ is given by the linearly independent basis $a_{11}, \cdots, a_{r s}$ modulo $M$ of $M^{*} / M$. Each $g \in G$ defines a linear transformation $f(g)$ on the module of $(r, s)$. matrices over $M^{*}$ as follows: If $\left(x_{11}, \cdots, x_{r s}\right) \rho_{1}(g)=\left(y_{11}, \cdots, y_{r s}\right)$, then $\left(x_{i j}\right) \cdot f(g)=\left(y_{i j}\right)$. We define also an $(r, s)$-matrix $c(g)$ over $M$ by the relation $N(c(g))=N(0)^{g}$. If $b$ and $b^{\prime}$ are such that $N(b)^{g}=N\left(b^{\prime}\right)$, then we have $\left(a_{i j}+b_{i j}\right)^{g}=\left(a_{i j}+b_{i j}^{\prime}\right) \cdot f(g)$. Since $\left(a_{i j}\right)^{g}=\left(a_{i j}+c(g)\right) \cdot f(g)$, we see that $b^{\prime}=c(g)+b^{g} \cdot f(g)^{-1}$. Thus:

$$
N(b)^{g}=N\left(c(g)+b^{g} \cdot f(g)^{-1}\right) .
$$

If we apply this formula to the case where $b=c(h)$ with $h \in G$, then we have

$$
c(h g)=c(g)+c(h)^{g} \cdot f(g) \cdot .
$$

Now, let $g_{1}, \cdots, g_{t}$ be such that $G=\sum G_{0} g_{i}$ and we set $d=t^{-1}\left(\sum c\left(g_{i}\right)\right)$. We want to show that $N(d)^{g}=N(d)$ for every $g \in G$. Indeed, $c(g)+d^{g} \cdot f(g)^{-1}=c(g)+t^{-1}\left(\sum c\left(g_{i}\right)^{g} \cdot f(g)^{-1}\right)=c(g)+t^{-1}\left(\sum\left(c\left(g_{i} g\right)-c(g)\right)=\right.$ $t^{-1}\left(\sum c\left(g_{i} g\right)\right)=d .^{5)} \quad$ Therefore $M^{*}=M \oplus N(d)$ as a representation module of $G$, which completes the proof.

4) The formula (1) below shows that $N(b)$ is $G_{0}$-admissible if and only if $b^{g}=b \cdot f(g)$ for every $g \in G_{0}$.

5) Note that if $g^{\prime} \in G_{0} g$, then $c\left(g^{\prime}\right)=c(g)$, because $N(0)$ is a $G_{0}$-module. 
Corollary. If $p=0$, then the converse of the corollary to Lemma 5 is true, provided that the index $\left[G: G_{0}\right]$ is finite.

Lemma 7. Let $H$ be a subgroup of finite index of $G$. If a representation $\rho$ of $H$ in $G L(n, K)$ is not completely reducible, then the representation $\rho^{*}$ of $G$ induced by $\rho$ is not completely reducible.

Proof. Let $M$ be the representation module of $\rho$. $M$ contains an $K$-H-module $N$ which is not a direct summand of $M$. Let $M^{*}$ be the representation module of $\rho^{*}$. Then $M^{*}$ is of the form $M \oplus \sum M g_{i}$ where $g_{i}$ are such that $G=H+\sum H g_{i}\left(g_{i} \notin H\right)$. It is obvious that $\sum M g_{i}$ is $H$-admissible. $M^{*}$ contains $N^{*}=N \oplus \sum N g_{i}$. If $N^{*}$ is a direct summand of $M^{*}$ as a $G-K$-module, then we have $M \oplus \sum M g_{i}=N \oplus \sum N g_{i} \oplus N^{\prime}$ as an $H$-K-module. Then we see that $M=N \oplus\left(M \cap\left(\sum N g_{i}+N^{\prime}\right)\right)$ as an $H$-K-module, which is a contradiction. Hence $N^{*}$ is not a direct summand of $M^{*}$ and $\rho^{*}$ is not completely reducible.

Corollary. If a finite group $G^{*}$ has order which is divisible by $p$, then $G^{*}$ has a representation which is not completely reducible.

Proof. $G^{*}$ has an element $a$ whose order is $p$. Then the subgroup $\left\{a^{i}\right\}$ is represented by $\left\{\left(\begin{array}{ll}1 & i \\ 0 & 1\end{array}\right)\right\}$, and we see the assertion by Lemma 7 .

Next we observe relationship between rational representations of a matric group $G$ and those of the closure of $G$.

Lemma 8. Let $G$ be a matric group and let $G^{*}$ be the closure of $G$. Let $\rho^{*}$ be a rational representation of $G^{*}$ and let $\rho$ be the restriction of $\rho^{*}$ on $G$. Then $\rho$ is irreducible if and only if $\rho^{*}$ is irreducible. $\rho$ is completely reducible if and only if $\rho^{*}$ is completely reducible.

Proof. $\rho(G)$ is dense in $\rho^{*}\left(G^{*}\right)$ and we see the assertions easily.

Lemma 9. Let $N$ be a normal subgroup of a matric group $G$ and let $\rho$ be an irreducible rational representation of $G$ into $G L(n, K)$, $K$ being a universal domain. If $N$ consists only of unipotent elements, then $N$ is contained in the kernel of the irreducible representation $\rho$. 
Proof. Since the set of all unipotent matrices in $G L(n, K)$ is closed, and since the image of a unipotent element under a rational representation is again unipotent, the closure $N^{*}$ of $\rho(N)$ consists only of unipotent elements. Therefore $N^{*}$ is nilpotent, hence is solvable. Therefore we may assume that every element $\left(a_{i j}\right)$ of $\rho(N)$ is such that $a_{i j}=0$ if $i>j$, whence $a_{i i}=1$ for every $i$. On the other hand, the corollary to Lemma 5 says that the restriction of $\rho$ on $N$ is completely reducible, whence $\rho(N)$ must consists only of the identity, which completes the proof.

3. The main result in the case where $G$ is connected and $p \neq \mathbf{0}$.

Theorem 1. Let $K$ be a universal domain of characteristic $p \neq 0$ and let $G$ be a connected matric group contained in $G L(n, K)$. Then the following three conditions are equivalent to each other:

( I ) Every rational representation of $G$ is completely reducible.

(II) $G$ is contained in a torus group, i.e., there is an element a of $G L(n, K)$ such that $a^{-1} G a$ is a subgroup of the diagonal group.

(III) The representation of $G$ by homogeneous forms of degree is completely reducible.

Proof. It is obvious by virtue of Lemma 8 that each of the above conditions for $G$ is equivalent to that for the closure of $G$. Therefore we may assume that $G$ is a connected algebraic linear group. It is well known that (II) implies (I) and it is obvious that (I) implies (III). Thus we have only to show that (III) implies (II). Assume that (III) is true and that (II) is not true, and we shall lead to a contradiction. Lemma 3 shows that $G$ contains an element $g$ which is not semi-simple. Then the unipotent part $g_{u}$ of $g$ is different from the identity and is contained in $G$ (cf. Borel's paper), hence $G$ contains a connected closed unipotent subgroup $U \neq 1$ by Lemma 2 . The representation module $F_{p}$ of the representation $\rho_{p}$ of $G$ by homogeneous forms of degree $p$ is nothing but the module of homogeneous forms of degree $p$ in $n$ variables $X_{1}, \cdots, X_{n}$ on which element $g$ of $G$ operates by the rule $h\left(X_{1}, \cdots\right.$, $\left.X_{n}\right)^{g}=h\left(\left(X_{1}, \cdots, X_{n}\right) g\right) . \quad F_{p}$ contains $M=\sum X_{i}^{p} K$, which is also a 
representation module of $G$. Hence (III) implies that $M$ is a direct summand of $F$. Thus $F_{p}=N \oplus M$. For each monomial $n_{i_{1} \cdots i_{n}}=$ $X_{1^{1}} \cdots X_{n}^{i_{n}}$ with $i_{j}$ such that $i_{j}<p$ and $\sum i_{j}=p$, there is a uniquely determined element $m_{i_{1} \cdots i_{n}}$ of $M$ such that $f_{i_{1} \cdots i_{n}}=n_{i_{1} \cdots i_{n}}+m_{i_{1} \cdots i_{n}}$ form a linearly independent basis for $N$. We note that $N$ and $M$ are representation modules of $U$. Hence we have only to show that:

The decomposition $F_{p}=N \oplus M$ as a repersentation module of the connected closed unipotent group $U$ lead us to a contradiction.

Let $u=\left(u_{i j}\right)$ be a generic point of $U$ over the universal domain $K$. We may replace $U$ with a conjugate of $U$. Hence we may assume first that $u_{i j}=0$ if $i>j$, whence $u_{i i}=1$ for each $i$. Set $K^{*}=K\left(\left\{u_{i j}^{p}\right\}\right)$, and we choose $(k, l)$ so that $u_{k l} \notin K^{*}, u_{i j} \in K^{*}$ if $i>k$ and such that $u_{k j} \in K^{*}$ if $j>l$. For each $A^{-1} U A$ ( $A$ being a triangular unipotent matrix), we can associate such an $(k, l)$, and we may assume that the pair $(k, l)$ for $U$ is lexicographically smallest among those $(k, l)$ for $A^{-1} U A$. Assume for a moment that there is a linear relation $\sum_{i} \alpha_{i} u_{k i} \in K^{*}$ with $\alpha_{i} \in K$ and $\alpha_{l} \neq 0$. We may assume that $\alpha_{l}=1$ and that $\alpha_{i}=0$ if $u_{k i} \in K^{*}$. Hence, in particular, $\alpha_{1}=\cdots=\alpha_{k}=\alpha_{l+1}=\cdots=\alpha_{n}=0$. Consider the unit matrix 1 and the matrix $c^{\prime}=\left(c_{i j}^{\prime}\right)$ such that (i) $c_{i j}^{\prime}=0$ if $j \neq l$, (ii) $c_{i \ell}^{\prime}=\alpha_{i}$ if $i \neq l$ and (iii) $c_{l l}^{\prime}=0$. Set $c=1+c^{\prime}$. Then obviously $c^{-1}=1-c^{\prime}$. Since $c^{-1} u \equiv u$ modulo $K^{*}$, we see easily that such an $(k, l)$ defined for $c^{-1} U c$ has the same $k$ and a smaller $l$ than our $(k, l)$, which is a contradiction. Therefore :

If $\alpha_{i} \in K$ and if $\alpha_{l} \neq 0$, then $\sum_{i} \alpha_{i} u_{k i} \notin K^{*}$.

Now, let $a=\left(a_{i j}\right)$ be an arbitrary element of $U$. Then $u a$ is also a generic point of $U$ over $K$. Since $u_{k j}(j>l)$ is in $K^{*}$, the $(k, j)$ component of $u a$ must be in $K^{*}$. This shows by virtue of (1) above that $a_{l j}=0$ if $j>l$. Since $a$ is arbitrary, we see that $u_{l j}=0$ for every $j \neq l$. Thus $X_{l}$ is $U$-invariant. Now we consider the elements $f_{i_{1} \cdots i_{n}}\left(i_{j}<p, \sum i_{j}=p\right)$. We denote by $g_{j}$ the element $f_{i_{1} \cdots i_{n}}$ such that $i_{j}=1, i_{l}=p-1$ for each $j=k, k+1, \cdots, l-1, l+1$, $\cdots, n$. Since we have

$$
\left(X_{k} X_{l}^{p-1}\right)^{u}=\sum u_{k j} X_{j} X_{l}^{p-1}
$$


we see that

$$
g_{k}^{u h}=\sum_{j \neq l} u_{k j} g_{j} .
$$

Consider the coefficient of $X_{l}^{p}$ in $g_{k}^{n}$; let it be $d$. (3) shows that $d$ is a linear combination of $u_{k j}(j \neq l)$ with coefficients in $K$. On the other hand, (2) shows that $d-u_{k l}$ must be in $K^{*}$. Thus we have a contradiction to (1) above, which completes the proof of Theorem 1.

\section{The main result in the case where $p=0$.}

Theorem 2. Let $K$ be a universal domain of characteristic $p=0$ and let $G$ be a matric group contained in $G L(n, K)$. Then the following conditions are equivalent to each other:

( I ) Every rational representation of $G$ is completely reducible.

(II) There is a normal snbgroup $G_{0}$ of finite index such that (i) $G_{0}$ is a subgroup of a torus group and (ii) the index of $G_{0}$ in $G$ is not divisible by $p$.

(III) The connected component $G_{0}$ of the identity of $G$ is a subgroup of a torus group and $\left[G: G_{0}\right]$ is not divisible by $p$.

If $G$ is an algebraic linear group, then the above conditions are equivalent to the following condition:

(IV) Every element of $G$ is semi-simple.

Proof. It is obvious that (III) implies (II) and that (II) implies (I) by virtue of Lemma 6 . Therefore, by Lemma 8 , we have only to prove the equivalence of (I), (III), (IV) in the case where $G$ is an algebraic linear group. Thus we assume that $G$ is algebraic, and let $G_{0}$ be the connected component of the identity of $G$. Assume first that (IV) is true. Then $G_{0}$ consists merely of semisimple elements, hence $G_{0}$ is a torus group by Lemma 3. If a semi-simple element $a$ has a finite order, then the order is prime to $p$. Therefore Lemma 4 implies that $\left[G: G_{0}\right]$ is not divisible by p. Thus (IV) implies (III). As we have remarked above, (III) implies (I). Assume now that (IV) is not true. Then, as we have seen in the proof of Theorem 1 , there is a unipotent element $u$ of $G$ which is different from the identity. If $u \in G_{0}$, then $G_{0}$ has a rational representation which is not completely reducible, hence 
$G$ itself has such one by Lemma 7 or by the corollary to Lemma 5 . If $u \notin G_{0}$, then the finite group $G / G_{0}$ has a representation which is not completely reducible, which is a rational representation of $G$. Thus we see that (I) is not true. Therefore (I) implies (IV), which completes the proof of Theorem 2 .

\section{The main result in the case where $p=0$.}

Theorem 3. Let $K$ be a universal domain of characteristic $p=0$ and let $G$ be a matric group contained in $G L(n, K)$. Then the following conditions are equivalent to each other:

( I ) Every rational representation of $G$ is completely reducible.

(II) The closure of $G$ has a faithful rational representation which is completely reducible.

(III) The radical of the closure of $G$ is a torus group.

Proof. It is obvious that (I) implies (II) by virtue of Lemma 8 . Lemma 9 shows that (II) implies (III). In order to show that (III) implies (I), we shall prove the following lemma:

Lemma 10. Let $G$ be a connected algebraic linear group and let $R$ be the radical of $G$. If $R$ is a torus group, then there is a closed connected normal subgroup $S$ such that (i) $G=R S$ and (ii) $R \cap S$ is a finite grovp. Furthermore, $R$ is contained in the center of $G$ (hence $R$ is the connected component of the identity of the center of $G)$.

Proof. For the fact that $R$ is contained in the center of $G$, see Borel's paper. Let $S$ be the subgroup generated by all unipotent elements of $G$. Then $S$ is obviously a normal subgroup. Each unipotent element is in a closed connected unipotent subgroup of $G$, hence $S$ is generated by closed connected subgroups, and therefore $S$ is a closed connected subgroup of $G$. Now, we may assume that $R$ is a diagonal group and that each $g \in G$ is given by

$$
g=\left(\begin{array}{cccc}
\rho_{1}(g) & \tau_{12}(g) & \cdots & \tau_{1 r}(g) \\
0 & \rho_{2}(g) & \cdots & \tau_{2 r}(g) \\
\cdots \cdots & \cdots \cdots \cdots \cdots \cdots \cdots \cdots \\
0 & \cdots & \cdots \cdots \cdots \cdots \cdots & \rho_{r}(g)
\end{array}\right)
$$


with irreducible representations $\rho_{1}, \cdots, \rho_{r}$. If $u \in G$ is unipotent, then $\rho_{i}(u)$ is unipotent, whence the determinant of $\rho_{i}(u)$ is 1 . Therefore we see that if $s \in S$, then the determinant of $\rho_{i}(s)$ is 1 . On the other hand, since $R$ is in the center of $G, \rho_{i}(R)$ is in the center of $\rho_{i}(G)$, hence by the famous lemma of Schur every element of $\rho_{i}(R)$ is of the form $k \cdot \rho_{i}(1)$ with $k \in K$. Therefore we see thet $R \cap S$ is a finite group. Since $S$ a is closed normal subgruop, $R S$ is a closed normal subgroup. Since $G / R$ is semi-simple, we see that $G / R S$ is semi-simple, unless $G=R S$. If $G \neq R S$, then $G / R S$ contains a non-trivial unipotent element, whence there must be a unipotent element of $G$ outside of $R S$, which is a contradiction to our construction of $S$. Therefore $G=R S$, which completes the proof.

Now we proceed with the proof of Theorem 3. By the corollary to Lemma 6 , we may assume that $G$ is connected. Lemma 8 allows us to assume that $G$ is an algebraic linear group. Let $R$ be the radical of $G$ and let $S$ be the normal subgroup given in Lemma 10. Since $R \cap S$ is a finite group and since $G=R S$, we see that $S$ is semi-simple, whence every rational representation of $S$ is completely reducible. Let $\rho$ be an arbitrary rational representation' of $G$. We may assume that $\rho(R)$ is a diagonal group, whence the complete reducibility of the restriction of $\rho$ on $S$ implies the complete reducibility of $\rho$, which completes the proof.

\section{Another result.}

Let $K$ be a universal domain of an arbitrary characteristic and let $G$ be a connected algebraic linear group with universal domain $K$, throughout this section.

Theorem 4. Every rational representation of $G$ is completely reducible if (and only if) the following is true:

If $\rho^{\prime \prime}=\left(\begin{array}{cc}1 & \lambda \\ 0 & \rho^{\prime}\end{array}\right)$ is a rational representation of $G$, then $\rho^{\prime \prime}$ is equivalent to the representation $\left(\begin{array}{ll}1 & 0 \\ 0 & \rho^{\prime}\end{array}\right)$.

Proof. Let $\rho=\left(\begin{array}{ll}\rho_{1} & \tau \\ 0 & \rho_{2}\end{array}\right)$ be a rational representation of $G$. We 
have only to show that $\rho$ is equivalent to the representation $\left(\begin{array}{ll}\rho_{1} & 0 \\ 0 & \rho_{2}\end{array}\right)$. Since $\rho(a b)=\rho(a) \rho(b)$, we have

$$
\tau(a b)=\rho_{1}(a) \tau(b)+\tau(a) \rho_{2}(b) \text { for any } a, b \in G .
$$

Let $x$ be a generic point of $G$ over $K$ and we consider $f(x)=$ $\tau(x) \rho_{2}(x)^{-1} . \quad f(a)$ is then well defined for any $a \in G$. The relation (1) implies that $f(a b)=\rho_{1}(a) \tau(b) \rho_{2}(b)^{-1} \rho_{2}(a)^{-1}+\tau(a) \rho_{2}(a)^{-1}=$ $\rho_{1}(a) f(b) \rho_{2}(a)^{-1}+f(a)$ for any $a, b \in G$, whence

$$
f(x a)=\rho_{1}(x) f(a) \rho_{2}(x)^{-1}+f(x) \text { for any } a \in G .
$$

Let $m, n$ be such that $\tau$ is an $(m, n)$-matrix and consider the module $L$ of all $(m, n)$-matrices over $K(x)$. Each element $g$ of $G$ defines an $K$-linear map $\phi_{g}$ on $L$ as follows :

$$
\phi_{g}\left(w_{i j}(x)\right)=\left(w_{i j}(x g)\right) .
$$

Thus $L$ becomes an $K$ - $G$-module. Let $M$ be the set of all $\rho_{1}(x) c \rho_{2}(x)^{-1}$ with $(m, n)$-matrices $c$ over $K$. Then $M$ is a finite $K$-module contained in $L$. Since $\rho_{1}(x a) c \rho_{2}(x a)^{-1}=\rho_{1}(x)\left(\rho_{1}(a) c \rho_{2}(a)^{-1}\right)$ $\times \rho_{2}(x)^{-1}(a \in G), M$ is $G$-admissible. Set $N=f(x) K+M$. Then the relation (2) shows that $N$ is also a finite $K-G$-module. We consider a representation $\rho^{*}$ of $G$ by the module $N$. The relation (2) shows that $f(x)$ is $G$-invariant modulo $M$, hence either $f(x) \in M$ or $\rho^{*}$ is equivalent to a representation of the form $\left(\begin{array}{ll}1 & \lambda \\ 0 & \rho^{\prime}\end{array}\right)$. The former case implies that $f(x)+\rho_{1}(x) c \rho_{2}(x)^{-1}=0$ with some $(m, n)$-matrix $c$ over $K$. By our assumption, the latter case implies that there is an element $\rho_{1}(x) c \rho_{2}(x)^{-1}$ of $M$ such that $f(x)+\rho_{1}(x) c \rho_{2}(x)^{-1}$ is $G$-invariant. Hence, in any case, there is an $(m, n)$-matrix $c$ over $K$ such that $f(x)+\rho_{1}(x) c \rho_{2}(x)^{-1}$ is $G$-invariant. Set $\tau^{*}=\tau-c \rho_{2}+\rho_{1} c$. Then, transforming $\rho$ by the matrix $\left(\begin{array}{cc}\rho_{1}(1) & c \\ 0 & \rho_{2}(1)\end{array}\right)$, we see that $\rho$ is equivalent to the representation $\left(\begin{array}{ll}\rho_{1} & \tau^{*} \\ 0 & \rho_{2}\end{array}\right)$. Set $f^{*}(x)=\tau^{*}(x) \rho_{2}(x)^{-1}$. Then $f^{*}(x)=f(x)-c+\rho_{1}(x) c \rho_{2}(x)^{-1}$, which is $G$-invariant by our choice of $c$. Therefore $f^{*}(x a)=f^{*}(x)$ for any $a \in G$, whence $f^{*}(x)$ $=f^{*}\left(x x^{-1}\right)=0$. This shows that $\tau^{*}=0$, which completes the proof of Theorem 4 . 
We note by the way that the matrix $f(x)$ has an interesting property as follows:

Proposition. Assume that $\rho=\left(\begin{array}{cc}\rho_{1} & \tau \\ 0 & \rho_{2}\end{array}\right)$ is a rational representation of $G$. Set $H=\{h ; h \in G, \tau(h)=0\}$. Then the homogeneous variety $G / H=\{g H\}$ is an affine variety, on which the coordinates of a point $g H$ are given by $f(g){ }^{6}{ }^{6}$

Proof. Since $\tau(1)=1$, the formula (1) in the above proof shows that $\tau\left(a^{-1}\right)=-\rho_{1}(a)^{-1} \tau(a) \rho_{2}(a)^{-1}$, hence $\tau\left(a^{-1} b\right)=\rho_{1}(a)^{-1}\left[\tau(b) \rho_{2}(b)^{-1}-\right.$ $\left.\tau(a) \rho_{2}(a)^{-1}\right] \rho_{2}(b)$. Therefore $f(a)=f(b)$ if and only if $a H=b H$, which proves the assertion.

6) If we consider the set of cosets $\mathrm{Hg}$, then it becomes an affine variety, on which the coordinates of $H g$ are given by $\rho_{1}(g)^{-1} \tau(g)$. 